\title{
In-line polarization controller for hollow core photonic bandgap fiber
}

\author{
Oliver Green, Xi Zhang*, Eric R. Numkam Fokoua, Meng Ding, Zitong Feng, Yong Chen, \\ Austin Taranta, Francesco Poletti, David John Richardson, Radan Slavík
}

the Optoelectronic Research Centre, University of Southampton, SO17 1BJ, UK

A R T I C L E IN F O

Keywords:

Hollow Core Fiber

Polarization Control

Optical Fiber

\begin{abstract}
A B S T R A C T
We demonstrate an in-line polarization controller for hollow core photonic bandgap fiber made by inserting a length of the fiber into a standard, off-the-shelf, 3-paddle controller as widely used for polarization control using standard single mode fibers. Although the operational principle of this 3-paddle controller is very different when using hollow core photonic bandgap fiber rather than SMF effective polarization control is readily achieved.
\end{abstract}

\section{INTRODUCTION}

Hollow core photonic band gap fibers (HC-PBGFs) have a number of intriguing optical properties compared with conventional silica glass-core optical fibers [1]. This is mainly thanks to the strongly reduced lightmatter interaction in the core which leads to ultralow optical nonlinearity, high damage thresholds, low latency, and a low propagation time and accumulated phase temperature sensitivity. These properties make such fibers of interest in applications such as laser beam delivery [2], optical fiber sensing [3], and optical communications [4]. For practical uses, these fibers must be efficiently inter-connected with existing optical components such as splitters and isolators or, alternatively, these components should be made directly from HC-PBGF. One such component is the in-line optical polarization controller (PC), which is very often a critical component in fiber optic set-ups.

So far there have been two reports on making HC-PBGF PCs - either by twisting three fiber segments [5] or laterally squeezing three fiber segments [6]. Both of these methods require apparatus that is not routinely available in a usual fiber optic laboratory.

Here, we propose and test a new configuration of in-line HC-PBGF PC which uses the widely available, off-the-shelf, 3-paddle polarization controller designed for polarization control using standard single-mode optical fibers (SSMFs). We describe and illustrate how the principle of operation and function of the controller is different when using HC-PBGF rather than SSMF and demonstrate that effective polarization control is possible using our approach.

\section{POLARIZATION CONTROL PRINCIPLE}

A PC is a device that is capable of transforming polarized light of any input state of polarization (SOP) into an output with arbitrary SOP. Mathematically, the most straightforward realization of a PC is with three waveplates: two of them being quarter-wave plates (QWP) and the third one, that is inserted in between them, is a half-wave plate (HWP). It can be straightforwardly realized with bulk-optics waveplates, as sketched in Fig. 1 (a) where polarization control is achieved by rotating the individual waveplates. The first QWP is capable of generating a linearly-polarized beam from an input beam of arbitrary SOP. The HWP can then be used to rotate this linearly-polarized beam by any arbitrary angle. The second QWP then be rotated to perform the inverse function of the first QWP and to output a beam of any desired SOP.

To implement this principle in fiber optics using SSMF, it is necessary to generate the equivalent of a free space wave-plate but made of SSMF. Although straight and unperturbed SSMF has a very small birefringence, a controllable amount of birefringence can easily be created by squeezing or bending the fiber $[7,8]$. Of the many commercially available PCs the three paddle PC, shown in Fig. 1 (b), is perhaps the most common and frequently used. Here, lengths of fiber are bent into loops, typically of constant diameter, creating birefringence [8], and held in the paddles of the PC. Specific lengths of the fiber are bent into the paddles to create the QWPs and HWP, with the HWP needing double the length of bent. Waveplate rotation is then realized by rotating each of the paddles (analogous to the rotation of the plates in a free space embodiment), Fig. 1 (b). In practice, a very good level of arbitrary polarization control is achievable even if the length of the fiber in the loops is not exactly as designed.

As there is very weak interaction of light with the glass in HC-PBGF (as light propagates through the central hollow core), the polarization state is significantly less sensitive to bending or squeezing in a HC-PBGF as compared to SSMF [9]. Although this represents an advantage for most applications (i.e. providing greater immunity to external perturbations), it makes the task of making a PC from a HC-PBGF via externally induced birefringence more challenging. Fortunately, however, HC-PBGFs typically exhibit a moderate amount of birefringence due to slight 


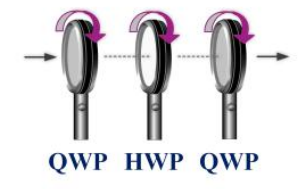

(a)

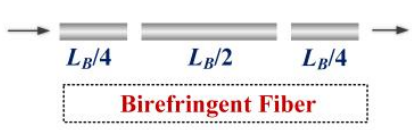

(c)

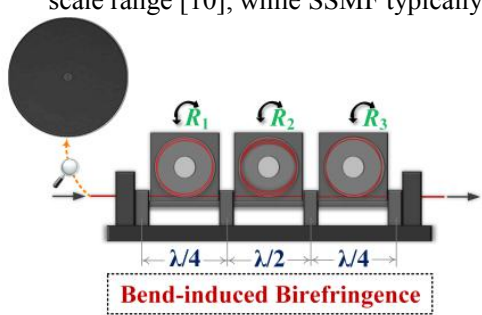

(b)

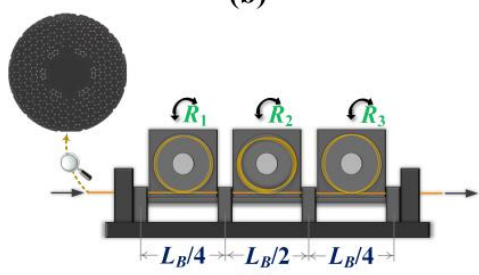

(d)

Fig. 1. Various implementations of polarization controller: (a) free space controller employing three waveplates that can be rotated; (b) SSMF-based controller employing three rotating fiber loops; (c) the principle of PC made from three segments of birefringent fiber $\left(L_{B}\right.$ is the beat-length), which we use for our HC-PBGF-made PC; (d) our HC-PBGF-made controller employing three rotating fiber loops.

length of tens of meters [11]. 19-cell HC-PBGF designs have a larger core than the 7-cell, and thus there is less interaction of light with the glass cladding, resulting in lower insertion loss [12] and a longer beat length in the range of tens of $\mathrm{cm}$ (Fig. 2).

We previously mentioned that a PC can be made in free space optics using three waveplates made of birefringent material. This concept can be translated into fiber optics: we can use three birefringent segments of optical fiber with their length being quarter of the beat-length (equivalent of QWP) and half beat-length (HWP), Fig. 1 (c). Although this in principle works with polarization-maintaining single-mode fibers, it would not be very practical: polarization control would require the ability to rotate each of the three fiber segments independently (analogous to the rotation of the free space components), whilst ensuring simultaneous alignment in between the segments to ensure low insertion loss.

Here, we propose to keep each required length of fiber isolated within one of the sections of a 3 paddle PC and to use the paddles to provide relative rotation of the "fiber waveplates". The fiber within the PC is fixed at four positions (at the beginning, after quarter beat-length, three quarters beat-length, and at the end at the full beat-length) to form the three segments of QWP, HWP, and QWP. Subsequently, we rotate each segment, creating twist at the four fixed points with the twist changing the axis of birefringence between the isolated lengths forming the three waveplates.

Having established the principle, let us discuss practical implementation with HC-PBGF. Keeping the entire length of the fiber within PC straight would result in a relatively long component (at least one beat-length, which is $\sim 40 \mathrm{~cm}$ for the 19-cell HC-PBGF we used, as we show later) and would require sophisticated components to rotate each segment. Once again, we will use the fact that HC-PBGF sensitivity to external perturbation (bend in this case) is relatively weak [9] and so we coil each of the lengths (corresponding to HWP or QWP) and put it into a standard commercial 3-paddle PC, Fig. 1 (d).

It is worth to re-iterate that although our HC-PBGF looks and functions like a commercially available three paddle PC for SSMF, its operational principle is very different: instead of bending the fiber to create the QWPs and HWP, the paddles are used to store the required lengths of intrinsically-birefringent fiber without inducing any significant additional birefringence.

\section{MEASUREMENT OF HC-PBGF BEAT-LENGTH}

The HC-PGF used in our experiments was an in-house made 19-cell HC-PBGF with a central air-hole diameter of $31 \mu \mathrm{m}$ and loss of 3.9 $\mathrm{dB} / \mathrm{km}$. The detailed HC-PBGF microstructure is shown in Fig. 3. To find the lengths of HC-PBGF corresponding to a QWP and HWP we needed to determine the HC-PBGF phase birefringence. We simulated this using a finite element method in Comsol Multiphysics [13], obtaining birefringence as difference between propagation constants of fundamental mode along the two axes of birefringence, Fig. 2. We see that the beatlength is expected to be $38 \mathrm{~cm}$ at $1550 \mathrm{~nm}$ and that it changes very little (within 35-40 cm) over a $50 \mathrm{~nm}$ bandwidth $(1520-1570 \mathrm{~nm}$ ). Subsequently, we measured the HC-PBGF phase birefringence. It is worth mentioning that in HC-PBGFs, the phase and group birefringence can be very different (by several orders of magnitude [14]), requiring care when choosing the right measurement method to ensure the phase birefringence 
is properly measured. We mention this as for standard polarizationmaintaining optical fibers, these two forms of birefringence are almost identical and are sometimes (mistakenly) interchanged in the literature.

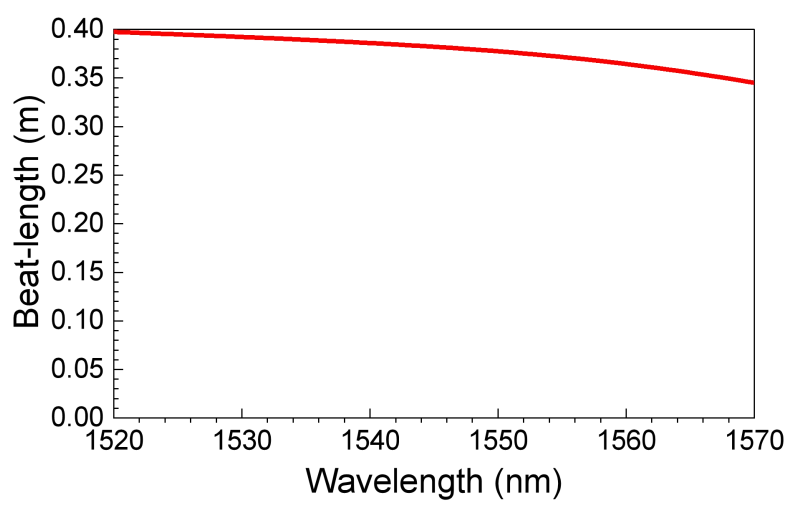

Fig. 2. Calculated beat-length of the used HC-PBGF as a function of wavelength.

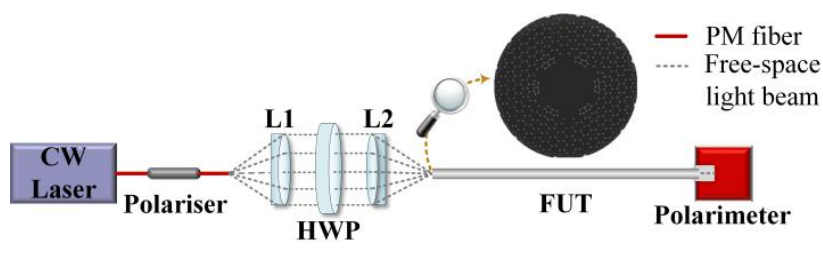

Fig. 3. Experimental setup of measuring HC-PBGF beat-length. $\mathrm{CW}$ Laser: Continuous Wave laser at $1550 \mathrm{~nm}$; L1: collimating lens; L2: focusing lens; FUT: fiber under test.

Our beat-length measurement is based on a cut-back method and is illustrated in Fig. 3. We use linearly polarized laser light at $1550 \mathrm{~nm}$, where the linear polarization plane can be changed via a free-space input HWP. Before launching light into the fiber under test (FUT), we analyzed the input light with a polarimeter (Thorlabs PAX1000IR2/M). By rotating the input HWP, the trace on the Poincare sphere went around the equator (where ellipticity $\chi$ is zero), exactly as expected for linearly polarized light as the angle of linear polarization is rotated.

Subsequently, we placed the FUT in between the input HWP and the polarimeter (adding a focusing lens to couple the light into the HC-PBGF), rotated the input HWP, and recorded the resulting trace on the Poincare sphere. For a FUT of half the beat-length (and its multiples), we should always get a linear polarization at the output, observed in our set-up as a trace going along the equator at the Poincare sphere as the linear polarization of the input light is rotated $(\chi=0)$. For a FUT with a length corresponding to a quarter of the beat-length (or $3 / 4,5 / 4, \ldots$ ), we should see a trace that follows a meridian on the Poincare sphere, passing through the North and South poles, which corresponds to circularly polarized light and $\chi=45^{\circ}$ maximum ellipticity). As shown in [15], maximum $\chi\left(\chi^{\max }\right)$ obtained when rotating the input HWP can be attributed to the phase difference between the principal axes of polarization of the FUT, with two extremes $\left(\chi^{\max }=0^{\circ}, \chi= \pm 45^{\circ}\right)$ described above.

We recorded $\chi^{\max }$ for a length of HC-PBGF and then chopped off a short length of it at the output, keeping the input (and thus also its polarization axis) intact and re-measured $\chi^{\max }$. We repeated this procedure (chopping off a short length of FUT and re-measuring) several times. The measured $\chi^{\max }$ as a function of the FUT length in shown in Fig. 4. As follows from [15], the relation between $\chi^{\max }$ and the phase difference between light propagating along the two FUT principal axes of polarization follows $\left|\sin \left(2 \pi L / L_{B}\right)\right|$. The absolute value is used as we always measure $\chi^{\max }$ as a positive number. The fit is shown in Fig. 4, resulting in beat-length $L_{B}=41 \mathrm{~cm}$, which is very close to the expected value of $38 \mathrm{~cm}$.

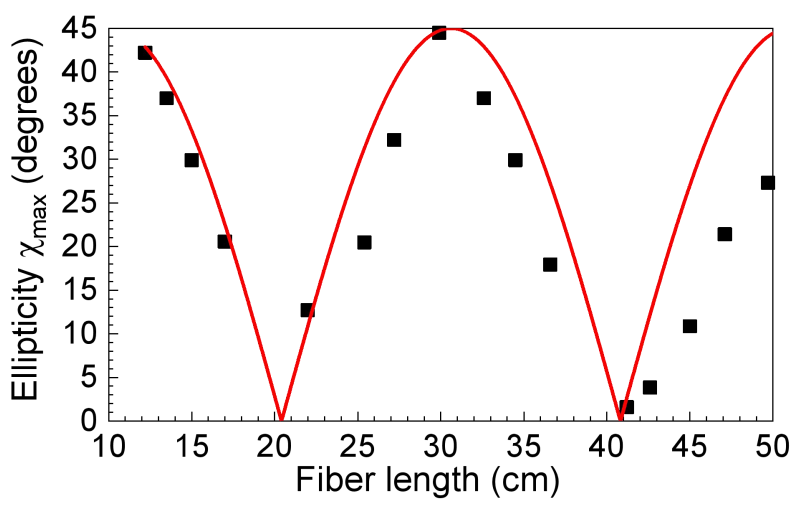

Fig. 4. Maximum ellipticity of output SOP achieved by rotating an input linear polarization state for various FUT lengths (dots) and theoretical fit (red line) giving $\boldsymbol{L}_{B}=\mathbf{4 1} \mathrm{cm}$.

\section{WAVEPLATES MADE OF HC-PBGF}

We have made two QWPs and a HWP by putting HC-PBGF segments into the paddles of a standard $3.5-\mathrm{cm}$ loop diameter polarization controller. To configure each specific paddle and to allow us to control the exact FUT that is manipulated, we unscrewed the other two. Details of our FUT arrangement for both waveplates are shown in Fig. 5. 
(a)

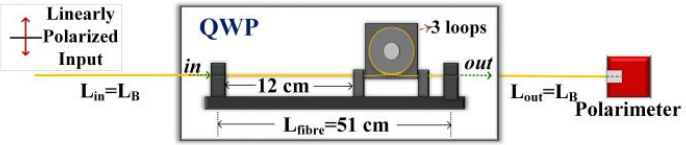

(b)

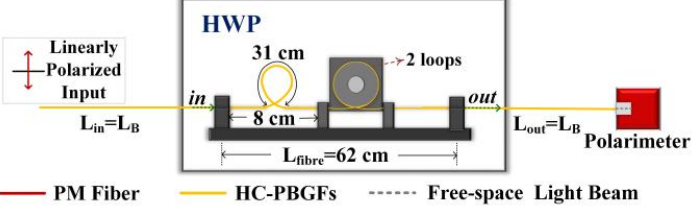

Fig. 5. Setup for characterization of the HC-PBGF-made waveplates: (a) QWP and (b) HWP. The linearly polarized input is identical to that shown in Fig. 3. The input and output pigtails are 1 beat-length long.

Given the fixed loop diameter of a standard 3-paddle PC and the two fiber fixing points (which are at the beginning and the end of the 3-paddle PC), we could not use exactly quarter $L_{B}(10.25 \mathrm{~cm})$ and half $L_{B}(20.5 \mathrm{~cm})$ HC-PBGF lengths, respectively. Instead, we used $1.25 L_{B}(51 \mathrm{~cm})$ and $1.5 L_{B},(62 \mathrm{~cm})$ which should provide the same behavior as quarter-wave $\left(0.25 L_{B}\right)$ and half-wave $\left(0.5 L_{B}\right)$ plates. Fig. 5 gives details on how we fixed the fiber inside the controller: we had 3 loops in QWP and 2 in HWP with the rest of the fiber was left free to move and rotate with the rotation of the paddles.

The experimental set-up used to characterize the performance of the HC-PBGF waveplates, Fig. 5, consisted of the same source and coupling optics as the set-up used to measure the beat-length (Fig. 3). The FUT sample in the PC was placed between two beat-lengths of the fiber. i.e. one beat-length HC-PBG placed at the input and one at the output. We chose this length as it should not alter the polarization state between the waveplate under study and the input and output, enabling us to know the polarization state at the beginning and the end of the fiber waveplate being characterized. As we rotated the paddle, the output SOP was analyzed using the polarimeter. (a)

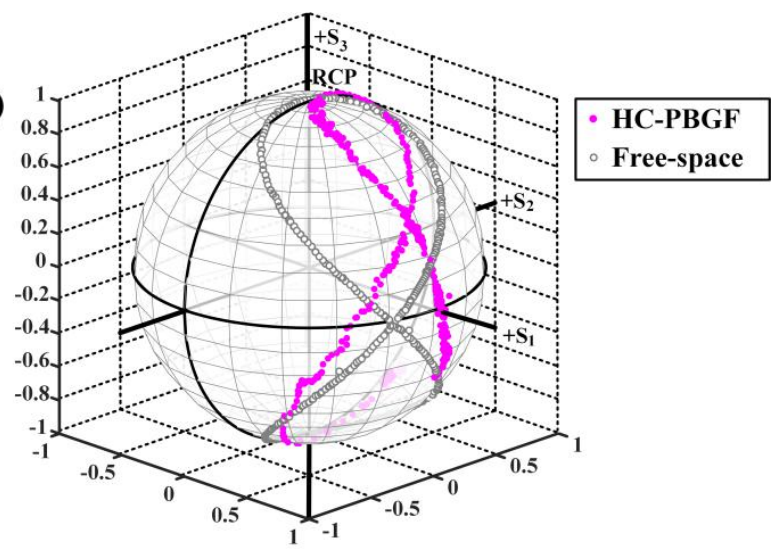

(b)

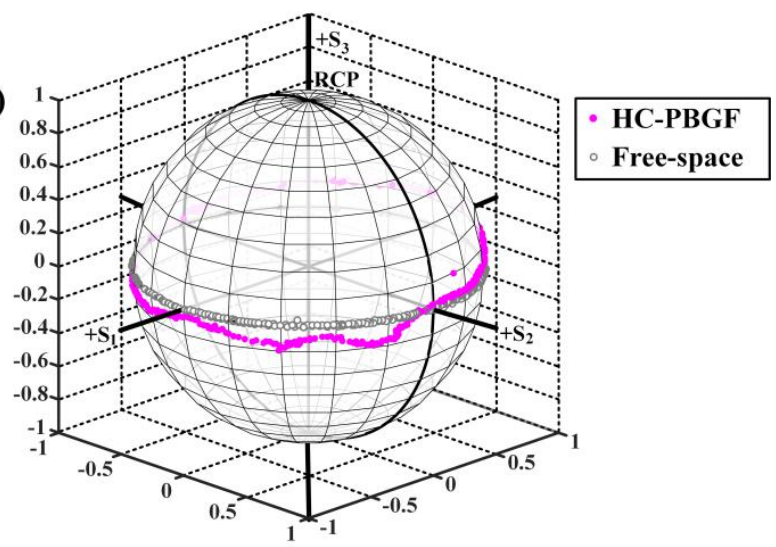

Fig. 6. Evolution of the measured output SOP on the Poincare sphere for (a) QWP, and (b) HWP. The solid dots: measured SOP evolution of HCPBGF-made plates as the paddle was rotated from one side to the other; the circles: measured SOP evolution of a free-space waveplate.

Fig. 6 (a) and (b) show the evolution of the output SOP of a HC-PBGF QWP and HWP represented by the solid dots when we rotated the paddle from one side to the other, respectively. For comparison, we also replaced the FUT waveplates with free-space QWP and HWP, shown as the traces composed of empty circles in the figures. The trajectory of the HC-PBGF QWP in Fig. 6 (a) follows the expected figure of " 8 " shape from the North Pole (right-handed circular polarization) to the South Pole (left-handed circular polarization) passing the equatorial plane (linear polarization) of the Poincare sphere. Meanwhile the trajectory of the HC-PBGF HWP in Fig. 6 (b) follows the equator, similarly to a free-space HWP. We believe the differences in the output SOP traces between the HC-PBGF waveplates and free-spaces waveplates are mainly due to the stress in the fiber induced when we rotated the paddles.

It is worth mentioning that the traditional 3-paddle SSMF-based polarization controller also shows small deviations from the expected behaviour for each of its paddles, as demonstrated in Fig. 7. To get these results, we used a single paddle with 2 and 3 loops of SSMF for QWP and HWP, as instructed by the paddles manufacturer (Thorlabs). 

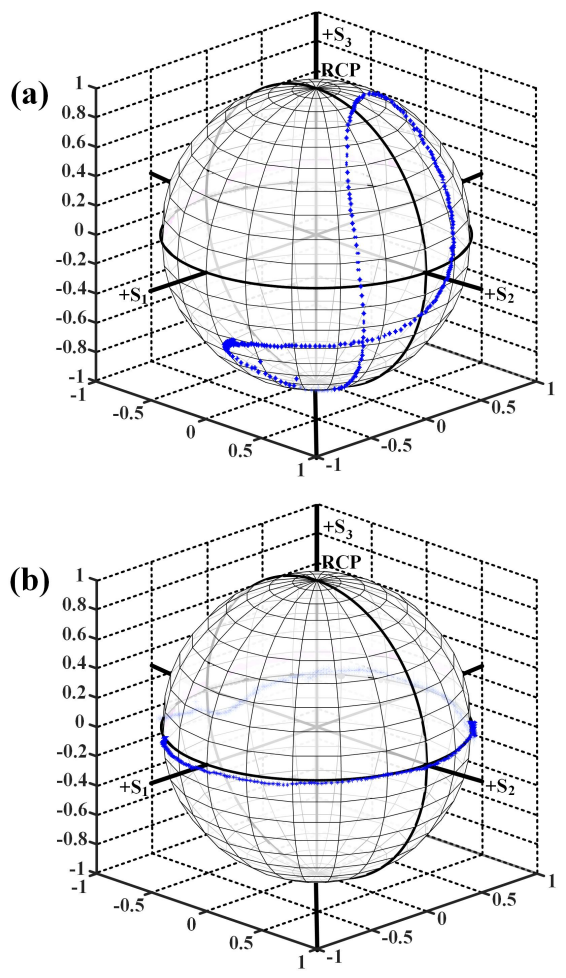

Fig. 7. Evolution of the measured output SOP on the Poincare sphere for SSMF-made single-paddle (a) QWP, and (b) HWP . The paddle was rotated from one side to the other.

\section{HC-PBGF POLARIZATION CONTROLLER}

We re-assembled our 3-paddle PC and constructed an all-HC-PBGF PC by taking a length of HC-PBGF and making a single HC-PBGF loop in the first and third paddle (length close to quarter $L_{B}$ ) and two loops in the middle paddle (close to half $L_{B}$ ), Fig. 1 (d). Thus, the fiber lengths in the paddles were not exactly as required from the theory $(11$ and $22 \mathrm{~cm}$ in paddles as opposed to 10.25 and $20.5 \mathrm{~cm}$ corresponding to $L_{B} / 4$ and $L_{B} / 2$ ). Additionally, we expect the fiber twists in between the paddles to induce slightly distorted change to the polarization state in the fiber as compared to the pure polarization axis rotation considered. Hence, we expect some level of differences in the behavior as compared to theory. However, such a statement applies also to the 3-paddle controller made of SSMF (Fig. 7), and does not have any significant implications on its performance in most applications.

The output SOP of our HC-PBGF was measured using the same experimental system as shown in Fig. 5. For various input SOPs, we recorded the evolution of the output SOP on the Poincare sphere when we changed the three paddles, always achieving good coverage of the entire Poincare sphere.

\section{CONCLUSION}

We have suggested how to use a standard 3-paddle polarization controller designed for standard single-mode fiber with HC-PBGF. We reiterate that the loops in the paddles are not used to introduce birefringence, but rather to compactly store a length of the HC-PBGF and to allow its efficient rotation. We measured the beat-length of the HCPBGF used to be $41 \mathrm{~cm}$ and subsequently made quarter and halfwaveplates from it. We verified their operation using a polarimeter. Subsequently, we used quarter-half-quarter beat-length HC-PBGF segments in the 3-paddle polarization controller and tested its ability to cover the entire Poincare sphere for various input polarization states. Our simulations show that the HC_PBGF beat-length changes only very little over a $50 \mathrm{~nm}$ bandwidth and thus we expect the polarization controller to operate over a reasonably-large spectral range. Advantages of our implementation as compared to the state-of-the-art include the ability to use off-the-shelf components readily available in majority of fiber optics laboratories.

\section{Acknowledgements}

This work was supported by the UK EPSRC project "Airguide Photonics" (EP/P030181/1); R. Slavík was supported by an RAEng Senior Fellowship. The data in this paper is accessible through the University of Southampton research repository (DOI: 10.5258/SOTON/XXXX).

\section{REFERENCES}

[1] F. Poletti, M.N. Petrovich, and D.J. Richardson, "Hollow-core photonic bandgap fibers: technology and applications," Nanophotonics, vol. 2, no. 56, pp. 315-340, Dec. 2013.

[2] M. Michieletto, J. K. Lyngso, C. Jakobsen, J. Laegsgaard, O. Bang, and T. T. Alkeskjold, "Hollow-core fibers for high power pulse delivery," Opt Express, vol. 24, no. 7, pp. 7103-19, Apr. 2016.

[3] Z. Zhang, J. He, B. Du, K. Guo, and Y. Wang, "Highly sensitive gas refractive index sensor based on hollow-core photonic bandgap fiber," Opt Express, vol. 27, no. 21, pp. 29649-29658, Oct. 2019.

[4] F. Poletti, N.V. Wheeler, M.N. Petrovich, N. Baddela, E. Numkam Fokoua, J.R. Hayes, D.R. Gray, Z. Li, R. Slavík, and D.J. Richardson, "Towards high-capacity fibre-optic communications at the speed of light in vacuum," Nature Photonics, vol. 7, no. 4, pp. 279-284, Apr. 2013.

[5] M. Terrel, M. J. F. Digonnet, and S. Fan, "Polarization controller for hollow-core fiber," Optics Letters, vol. 32, no. 11, pp. 1524-1526, Jun. 2007.

[6] M. Pang and W. Jin, "A hollow-core photonic bandgap fiber polarization controller," Optics Letters, vol. 36, no. 1, pp. 16-18, Jan. 2011. 
[7] S. Rashleigh, "Origins and control of polarization effects in single-mode fibers," Journal of Lightwave Techology, vol. 1, no. 2, pp. 312-331, Jun. 1983.

[8] R. Ulrich, S. Rashleigh, and W. Eickhoff, "Bending-induced birefringence in single-mode fibers," Optics Letters, vol. 5, no. 6, pp. 273-275, Jun. 1980

[9] D. Chung, H.S. Park, F. Rotermund, and B.Y. Kim, "Measurement of bending-induced birefringence in a hollow-core photonic crystal fiber," Optics Letters, vol. 44, no. 23, pp. 5872-5875, Dec. 2019.

[10] M. Wegmuller, M. Legaré, N. Gisin, T.P. Hansen, Ch. Jakobsen, and J Broeng, "Experimental investigation of the polarization properties of a hollow core photonics bandgap fiber for $1550 \mathrm{~nm}$," Optics Express, vol. 13, no. 5, pp. 1457-1467, Feb. 2005.

[11] A. Galtarossa, L. Palmieri, M. Schiano, and T. Tambosso, "Measurements of beat length and perturbation length in long single-mode fibers," Optics Letters, vol. 25, no. 6, pp. 384-387, March 2000.

[12] R. Amezcua-Correa, N. Broderick, M. Petrovich, F. Poletti, and D. J. O. E. Richardson, "Design of 7 and 19 cells core air-guiding photonic crystal fibers for low-loss, wide bandwidth and dispersion controlled operation," Optics Express, vol. 15, no. 26, pp. 17577-17586, Dec. 2007.

[13] E. Numkam Fokoua, S.R. Sandoghchi, Y. Chen, G.T. Jasion, N.V. Wheeler, N.K. Baddela, J.R. Hayes, M.N. Petrovich, D. J. Richardson, and F. Poletti, "Accurate modelling of fabricated hollow-core photonic bandgap fibers," Optics Express, vol. 23, no. 18, pp. 23117-23132. Sept. 2015.

[14] J.M. Fini, J.W. Nicholson, B. Mangan, L. Meng, R.S. Windler, E.M. Monberg, A. DeSandtolo, F.V. DiMarcello, and K. Mukasa, "Polarization maintaining single-mode low-loss hollow-core fibres," Nature Communications, vol. 5, article 5085, Oct. 2014.

[15] A.V. Gladyshev, A.N. Kolyadin, A.F. Kosolapov, Yu.P. Yatsenko, A.D. Pryamikov, A.S. Biriukov, I.A. Bufetov, and E.M. Dianov, "Low-threshold $1.9 \mu \mathrm{m}$ Raman generation in microstructured hydrogen-filled hollow-core revolver fibre with nested capillaries," Laser Physics, vol. 27, no. 2, paper 025101, Dec. 2017. 